\title{
Limits on the optical magnitude of PSR 1821-24 in M28
}

\author{
F. K. Sutaria \\ Inter-University Centre for Astronomy and Astrophysics, Pune, India.
}

\begin{abstract}
The detection of a pulsed X-ray counterpart (RX J1824.22R52P) of the $3.05 \mathrm{~ms}$ pulsar PSR 1821-24, suggests the possibility of a part of the rotational energy loss of this high spindown rate pulsar being in the optical band. Archival HST data for M28 is used here to set upper limits on the optical V-band magnitude of PSR 1821-24. The optical limit extends the multiwavelength observations for this source and provides a constraint for theoretical models of pulsar emission.
\end{abstract}

Pulsed optical and high energy emission from pulsars can be attributed to either non-thermal acceleration of particles accelerated in the pulsar magnetosphere or to emission from the heated polar caps. Unpulsed optical emission can be caused by the thermal cooling of the neutron star. Both pulsed and unpulsed components are indirect probes of the poorly known conditions in the neutron star interior and of the neutron star equation of state (Pavlov et al., 1996). The isolated radio pulsar PSR1821-24 has a high spin down rate leading to rotational energy loss rate of $2.2 \times 10^{36} \mathrm{ergs} \mathrm{s}^{-1}$. This source has been detected as a rotation powered, non-thermal emitter of X-rays, having a luminosity $\left.L_{x}\right|_{0.1-2.4 \mathrm{KeV}}=1.6 \times 10^{33}(\mathrm{~d} / 5.1 \mathrm{kpc})^{2} \mathrm{ergs} \mathrm{sec}^{-2}$, by ROSAT HRI observations (Danner et al., 1997 and references therein). Since the spindown age of PSR 1821-24 is $\simeq 10^{7}$ years (Table 1), it can be expected to have a surface temperature $T<10^{5}{ }^{\circ} \mathrm{K}$ implying that the thermal emission would be predominantly in the far-UV to optical bands. Few old pulsars $\left(\tau \sim 10^{6}\right.$ years $)$ have been detected in the optical. Thus, an upper limit on the $\mathrm{V}$ band magnitude of PSR 1821-24 would add one more data point to this list, and a confirmed detection would constrain the amount of non-thermal optical emission from the neutron star magnetosphere.

Details of the observation parameters and data analysis are given in Sutaria (2000). Briefly, the data was obtained from a Sept. 1997 HST-WFPC2 exposure of $1120 \mathrm{~s}$. The radio position of PSR 1821-24 was obtained from the dense radiotiming observation of Cognard et al.(1996). Accounting for the proper motion of the pulsar, the co-ordinates of PSR1821-24 relative to the 1997 epoch are $\alpha=$ $18^{h} 24^{m} 32.0052^{s}$ and $\delta=-24^{\circ} 52^{\prime} 10^{\prime \prime} \cdot 7212$. The absolute astrometric accuracy of WFPC2 is only $\simeq 0.5$ arcsec, mainly because of inaccuracies of 0.5 " to 1 " in position of stars used in the Guide Star Catalog. Taking a lower estimate of $0.5^{\prime \prime}$ uncertainty, at least 8 optical sources have been detected in this region (Fig. 1). The photometry of these sources and their relative offsets has been quoted in Table 2. Of the eight candidates within the $0.5^{\prime \prime}$ astrometric "error circle", stars 4 and 5 are too bright to be considered for pulsar candidates. In order to determine whether these objects are foreground objects or not, photometric 


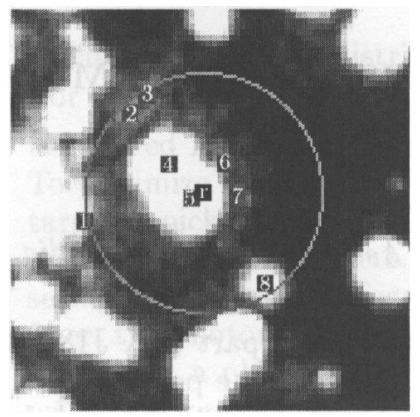

Table 1: A few relevant properties of PSR 1821-24. Fig.1 shows the positions of the sources detected within a circle of radius $0.5 "$, centered at the radio position of the pulsar, labelled ' $\mathrm{r}$ '.

\begin{tabular}{|ccc|}
\hline Property & units & Value \\
\hline Period $\left(P_{0}\right)$ & $\mathrm{s}$ & 0.00305 \\
$\dot{\mathrm{P}}$ & $\mathrm{s} / \mathrm{s}$ & $1.161 \times 10^{-18}$ \\
$\tau$ & $10^{6} \mathrm{yr}$ & 30 \\
Distance & $\mathrm{kpc}$ & 5.1 \\
\hline
\end{tabular}

Table 2. No. of counts for sources detected in a 0.5" field around the radio position of PSR1821-24. The offsets are calculated relative to the radio position of PSR 1821-24 (1997 epoch). All detections are at $3.5 \sigma$ level above the detection threshold.

\begin{tabular}{|ccccccc|}
\hline Star & $\alpha_{2000}$ & $\delta_{2000}$ & \multicolumn{2}{c}{ Offsets } & Total & STMAG \\
No & $(1824)$ & $(-2452)$ & $\Delta_{\alpha} \cos \delta$ & $\Delta_{\delta}$ & Counts & F555W \\
\hline 1 & 31.9756 & 10.998 & 0.402 & -0.2768 & 388.44 & 22.6 \\
2 & 32.0079 & 11.155 & -0.0375 & -0.4338 & 175.80 & 23.5 \\
3 & 32.0159 & 11.155 & -0.1455 & -0.4338 & 144.533 & 23.7 \\
4 & 32.0054 & 10.892 & -0.0027 & -0.1708 & 12227.12 & 18.9 \\
5 & 32.0022 & 10.741 & 0.0405 & -0.0198 & 14374.56 & 18.7 \\
6 & 32.0158 & 10.728 & -0.144 & -0.0068 & 363.38 & 22.72 \\
7 & 32.0118 & 10.599 & -0.144 & 0.1222 & 168.93 & 23.5 \\
8 & 31.9987 & 10.293 & 0.08835 & 0.4282 & 1083.147 & 21.5 \\
\hline
\end{tabular}

analysis in another band would be required. If the optical emission were entirely thermal in nature and hence strongly age dependent, PSR 1821-24 would be too faint to be detected in this 1120s exposure (Sutaria, 2000). Even assuming that optical component is enhanced by non-thermal emission, PSR1821-24 is unlikey to be as bright as any of the other 6 sources in table 2 because of its age and distance, thus implying that a visual band magnitude of 23.7 in STMAG system is only an upper limit on the optical counterpart for this source.

\section{References}

Cognard, I., Bourgois, G., Lestrade, J.-F., Biraud, F., Aubry D., Darchy, B., Drouhin, J. P., 1996, A\&A, 311, 179

Danner, R., Kulkarni S.R., Saito, Y., Kawai, N., 1997, Nature, 388, 751

Pavlov,G.G. , Stringfellow,G.S., Córdova, F.A., ApJ467, 370-384, (1996).

Sutaria, F. K., 2000, in preparation for ApJ 